\title{
SINGULAR SOBOLEV CONNECTIONS WITH HOLONOMY
}

\author{
L. M. SIBNER AND R. J. SIBNER
}

We consider local Sobolev connections on $S U(2)$ bundles over the complement, in $R^{4}$, of a smoothly embedded compact 2-manifold. Finite action implies that a holonomy condition is satisfied and we obtain an a priori estimate for the connection 1-form in terms of curvature and the flat connection carrying the holonomy. The a priori estimate classifies the possible singularities in these connections by the set of flat connections. In a certain case, this leads to smoothness and extendability results.

Let $N$ be a full 4 -dimensional neighborhood of the singular set $S$. The objects of study are connections $D=d+A$ defined on $S U(2)$ bundles over $X=N \backslash S$. We assume that $A \in H_{1, \text { loc }}^{2}(X)$ and that the action is finite, i.e., the curvature $F=d A+A \wedge A$ is in $L^{2}(N)$.

The following holonomy condition was first stated by Cliff Taubes. Choose coordinates $(r, \theta, u, v)$ with $(u, v)$ coordinates on $S$ and $(r, \theta)$ coordinates in a plane normal to $S$. Fixing $u$ and $v$, and denoting by $A_{\theta}$ the $\theta$ component of $A$, the initial value problem for an $S U(2)$ valued function,

$$
\frac{d g_{r}}{d \theta}+A_{\theta} g_{r}=0, \quad g_{r}(0)=I,
$$

has a unique solution $g_{r}(\theta)$, with $g_{r}(2 \pi)=J_{r} \in S U(2)$. The holonomy condition we require is

$$
\lim _{r \rightarrow 0} J_{r}=J^{\mathrm{b}} \text { exists. }
$$

This condition is gauge invariant up to conjugacy in $S U(2)$. Our results can be formulated in two theorems.

THEOREM 1. If $A$ and $F$ are smooth on $N \backslash S$ and $F \in L^{2}(N)$, then $(\mathrm{H})$ is satisfied for almost all $u$ and $v$. Up to conjugacy, the limit is independent of $u$ and $v$.

Next, assume $(\mathrm{H})$ holds. Locally, the conjugacy class $\left[J^{b}\right] \in S U(2)$ uniquely defines a flat connection $A^{b}=C d \theta$ with $C$ a constant element of $s u(2)$ determined up to a similarity transformation. Our second result uses holonomy to obtain an a priori estimate. We denote by $X_{0}$ and $N_{0}$ the intersections of $X$ and $N$ with a small open set in $R^{4}$ having nonvoid intersection with $S$.

Received by the editors February 23, 1988.

1980 Mathematics Subject Classification (1985 Revision). Primary 35J60, 53C80.

Research of the first author partially supported by NSF grant DMS-8501419.

Research of the second author partially supported by NSF grant INT-8411481. 
THEOREM 2. Suppose $\hat{D}=d+\hat{A}$ satisfies $(\mathrm{H})$ with $\hat{A} \in H_{1, \mathrm{loc}}^{2}\left(X_{0}\right)$ and $\|F\|_{L^{2}\left(N_{0}\right)}$ sufficiently small. Then there is a flat connection $A^{b}$ determined by $\left[J^{b}\right]$, and a universal constant $K$, such that $\hat{D}$ is gauge equivalent to $D=d+A$, with $d^{*} A=0$ and

$$
\left\|A-A^{\mathrm{b}}\right\|_{H_{1}^{2}\left(N_{0}\right)} \leq K\|F\|_{L^{2}\left(N_{0}\right)} .
$$

Note that if $\left[J^{b}\right]=I$, then $A$ is gauge equivalent to the zero connection form. In this case, $D$ extends as an $H_{1}^{2}$ connection to all of $N_{0}$. If, in addition, field equations are satisfied, more smoothness follows from elliptic theory.

Theorem 1 is proved by making a good choice of gauge in which the Fourier coefficients of $A_{\theta}$ can be estimated in terms of $F$. These estimates can be used to show that $A_{\theta} d \theta$ converges to a flat connection as $r$ tends to zero. This flat connection carries the holonomy. To show that the limit is independent of $u$ and $v$ requires another good choice of gauge and Stokes' theorem.

To prove Theorem 2, we carry out a plan of attack suggested by Cliff Taubes. This involves an open-closed argument similar to that used in $\left[\mathbf{U}_{1}\right.$, Theorem 1.3]. The large space consists of the appropriate Sobolev space of connections satisfying the same holonomy condition. This space is shown to be connected. The subspace consists of connections which admit a Hodge gauge satisfying certain boundary and limiting conditions which imply the a priori estimate. (Detailed proofs will appear in a forthcoming article.)

Theorem 1 settles a conjecture of Atiyah's. Both theorems are related to recent work on the moduli space of magnetic monopoles over hyperbolic 3space $[\mathbf{A}, \mathbf{B}, \mathbf{C}, \mathbf{F}]$ and to Yang-Mills fields over $S^{4}$ whose topological charge is not integral $\left[\mathbf{F H}_{1}, \mathbf{F H}_{2}\right]$.

ACKNOWLEDGEMENTS. We are greatly indebted to Cliff Taubes and Ed Miller for their invaluable assistance and encouragement.

\section{REFERENCES}

[A] M. F. Atiyah, Magnetic monopoles in hyperbolic spaces, Vector Bundles on Algebraic Varieties, Tata Institute of Fundamental Research, Bombay (1984), 1-33.

[B] P. J. Braam, Magnetic monopoles on three-manifolds, J. Differential Geom. (to appear).

[C] A. Chakrabarti, Spherically and axially symmetric $S U(N)$ instanton chains with monopole limits, Nuclear Physcis B248 (1984), 209-252.

[F] A. Floer, Monopoles on asymptotically euclidean 3-manifolds, Bull. Amer. Math. Soc. (NS) 16 (1987), 125-127.

[FH F $_{1}$ P. Forgacs, Z. Horvath and L. Palla, An exact fractionally charged self dual solution, Phys. Rev. Lett. 46 (1981), 392.

$\left[\mathbf{F H}_{2}\right]-$, One can have noninteger topological charge, Z. Phys. $C$-Particles and Fields 12 (1982), 359-360.

[S] L. M. Sibner, Removable singularities of Yang-Mills fields in $R^{3}$, Compositio Math. 53 (1984), 91-104.

[Sm] P. D. Smith, Removable singularities for the Yang-Mills-Higgs equations in two dimensions, preprint.

[SS] L. M. Sibner and R. J. Sibner, Removable singularities of coupled Yang-Mills fields in $R^{3}$, Comm. Math. Phys. 93 (1984), 1-17.

$\left[\mathbf{U}_{1}\right]$ K. Uhlenbeck, Connections with $L^{p}$ bounds on curvature, Comm. Math. Phys. 83 (1982), 31-42. 
$\left[\mathbf{U}_{2}\right] \ldots$, Removable singularities in Yang-Mills fields, Comm. Math. Phys. 83 (1982), 11-29.

Department of Mathematics, PolytechniC University of NeW York, BROOKLYN, NEW YORK 11201

Department of MATHEMATiCs, BRooklyn College (CUNY), Brooklyn, NEW YORK 11210 
